\title{
Study on the dynamic performance of concrete mixer's mixing drum
}

\author{
Jiapeng Yang ${ }^{1}$, Hua Zeng ${ }^{2}$, Tongqing $\mathrm{Zhu}^{2}$, and Qi An ${ }^{1}$ \\ ${ }^{1}$ School of Mechanical and Power Engineering, East China University of Science and Technology, Shanghai, \\ 200237, People's Republic of China \\ ${ }^{2}$ Shanghai Electric Hydraulics \& Pneumatics Co., Ltd., Shanghai, 201100, People's Republic of China
}

Correspondence to: Qi An (anqi@ecust.edu.cn)

Received: 2 December 2016 - Revised: 11 March 2017 - Accepted: 17 May 2017 - Published: 16 June 2017

\begin{abstract}
When working, the geometric distribution shape of concrete in concrete mixing truck's rotary drum changes continuously, which cause a great difficulty for studying the dynamic performance of the mixing drum. In this paper, the mixing system of a certain type of concrete mixing truck is studied. A mathematical formulation has been derived through the force analysis to calculate the supporting force. The calculation method of the concrete distribution shape in the rotary drum is developed. A new transfer matrix is built with considering the concrete geometric distribution shape. The effects of rotating speed, inclination angle and concrete liquid level on the vibration performance of the mixing drum are studied with a specific example. Results show that with the increase of rotating speed, the vibration amplitude of the mixing drum decreases. The peak amplitude gradually moves to the right with the inclination angle increasing. The amplitude value of the peak's left side decreases when tilt angle increases, while the right side increases. The maximum unbalanced response amplitude of the drum increases with the decrease of concrete liquid level height, and the vibration peak moves to the left.
\end{abstract}

1

\section{Introduction}

The rotary drum of Concrete mixing truck is a special hollow rotor, the section geometry of which is complicated. The calculation of supporting force for the drum is difficult due to the inclined installation and the changing concrete state. With the rotation of the drum, the shape of concrete will change, which lead to the complex vibration performance.

At present, the research on the mechanical and vibration performance of concrete mixing drum is still under development. Li et al. (2013) studied the changing loads at both supporting points with different conditions including uphill, downhill, uniformly driving, full load, no-load, emergency brake, etc. The test method is used to verify whether the static mechanical performance of the reducer meets the requirements of drum's application. Y. D. Gao et al. (2013) analyzed the mechanical properties of the mixing drum's front supporting by using software ANSYS. The effect of different exciting forces on drum's natural frequency and vibration mode is studied in detail. An improved scheme of the front support- ing structure with good performance is presented. Yan (2013) established the virtual simulation model of the mixing truck by the ADAMS software, and investigated the working principle of the mixing drum. The dynamic performance of the vehicle is studied under three conditions of the linear braking, the curve driving and the curve braking.

The rotary drum of concrete mixing truck is a kind of typical tilt rotor, the dynamic characteristics of which is different from the rotors working in horizontal state. Research on the dynamic properties of concrete mixer's rotating drum is not enough in the world. The existing literatures on the dynamic performance of the bearing rotor system are mainly aimed at horizontally placed rotors. Matthew and Sergei (2015) and Shi et al. (2013) studied the dynamic characteristics of horizontal and vertical rotor system supported by journal bearing, and analyzed the effects of bearing elasticity on the orbit of bearing center. Y. Gao et al. (2013) and Harsha (2005) made some researches on rotor's vibration properties supported by rolling element bearings with taking bearing structure, installation errors into consideration. Liu et al. (2012) 


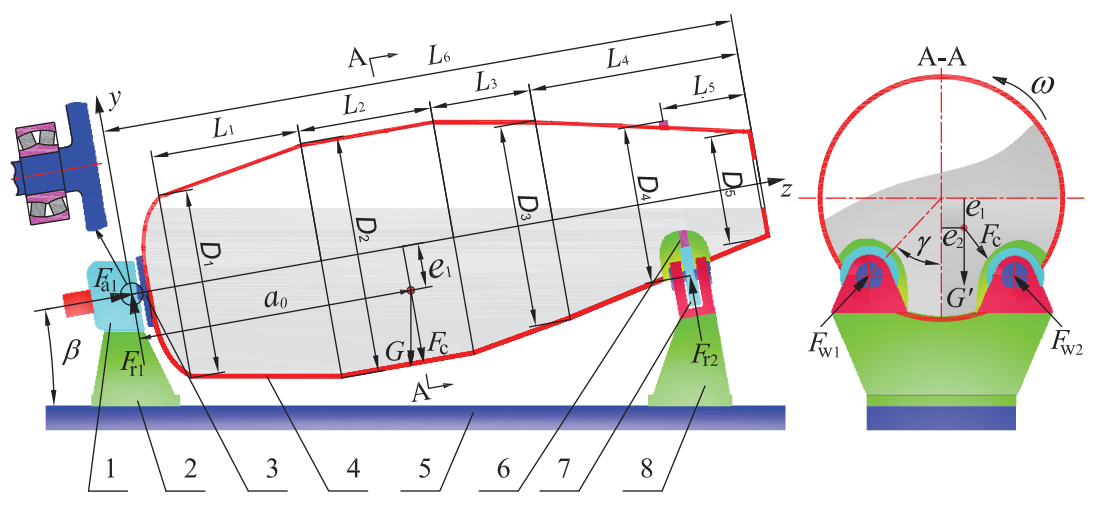

Figure 1. Structure and mechanics analysis of the concrete mixer system. 1: gear reducer, 2: front bracket, 3: flange, 4: mixing drum, 5: subframe, 6: raceway, 7: rolling wheel 8: rear bracket.

and Qian et al. (2011) built the dynamic equations of the inclined rotor supported by journal bearing by the use of finite element method, and the equations are solved by Newmark method (Dukkipati et al., 2000). The influences of tilting angle with different loads on the vibration performance of the system are analyzed in detail. Misaligned contact occurs even for taper roller bearings because of different contact conditions between rollers and inner and outer raceways as well as with the retaining rib. Therefore, all rotors run with some degree of misalignment (Rahnejat and Gohar, 1979). Kabus et al. (2014) dealt with the same issue as Rahnejat and Gohar (1979), where numerical analysis of non-Hertzian tilted rollers to races contacts are required for a misaligned rotorbearing system. Aleyaasin et al. (2000) and Tsai and Huang (2013) used transfer matrix method to establish the dynamic model for rotor-bearing system with multiple degrees of freedom. The critical speed, unbalanced response and vibration mode are studied.

It can be found through the analysis of literatures that although some researches have studied bearing-rotor system, the studies on the dynamic performance of concrete mixing truck's rotary drum are few. In this paper, a type of concrete truck's mixing drum is taken as the research object. The calculation method of supporting force is established on the base of mechanics theory. The computational model of the concrete shape in the rotary drum is implemented. A new transfer matrix is built to calculate the dynamic performance. With a specific example, the dynamic response of the mixing drum is studied numerically.

\section{Mechanics analysis of mixing drum}

The mixing system structure and force analysis of concrete mixing truck are shown in Fig. 1. The mixing drum is connected to the gear reducer by flange, and the gear reducer is installed on the front bracket. The right side of the drum is supported by two rolling wheels that are mounted on the rear bracket. The wheels are installed symmetrically. Each wheel is supported by a pair of tapered roller bearings. The centers of the two wheels and the drum center form an angle $2 \gamma$. The whole rotating drum is inclined, and the angle of the installation is $\beta$ which can be adjusted by a machine frame.

The mixing drum is subjected to gravity $G$, centrifugal force $F_{\mathrm{c}}$ and the supporting forces of the roller wheels and the bearing in gear reducer. Different from the horizontal rotor, the gravity and centrifugal force of the drum are not in the same plane when the drum rotates. As shown in Fig. 1, the forces on the left are radial force $F_{\mathrm{r} 1}$ and axial force $F_{\mathrm{a} 1}$. The roller wheels do not bear the axial force, so the gravity component of the internal drum and its concrete along the axial direction is borne entirely by the bearing in gear reducer. And the force on the right includes $F_{\mathrm{r} 2}$ by roller wheels $\left(F_{\mathrm{r} 2}\right.$ is the resultant force of $F_{\mathrm{w} 1}$ and $F_{\mathrm{w} 2}$ ).

Before the mechanics analysis of the mixing drum, the following assumptions are introduced: (a) the inner ring bearing and the flange are tight fitted (it is true in actual installation), and relative sliding does not occur between flange and inner ring. (b) Machining and installing error of bearing housing are neglected. (c) There is no bearing clearance error, and roller-raceway contact is in the range of elastic deformation and under elastohydrodynamic conditions. (d) The rotating drum can be regarded as a hollow shaft, and the shape of the section and the geometric size of the cross section are not change when the elastic deformation occurs. (e) The axial deformation and the geometric error of the drum are ignored.

As Fig. 1 shows, according to the force balance condition, the force balance equations of the mixing drum can be obtained:

$$
\left\{\begin{array}{l}
F_{\mathrm{r} 1}+F_{\mathrm{r} 2}-\left(G^{2} \cos ^{2} \beta+F_{\mathrm{c}}^{2}+2 e_{1} G F_{\mathrm{c}} \cos \beta / \sqrt{e_{1}^{2}+e_{2}^{2}}\right)^{1 / 2}=0 \\
a_{0} F_{\mathrm{r} 1}+\sqrt{e_{1}^{2}+e_{2}^{2}} G \sin \beta-F_{\mathrm{r} 2}\left(L_{6}-L_{5}-a_{0}\right)=0 \\
F_{\mathrm{w} 1} \cos \gamma+F_{\mathrm{w} 2} \cos \gamma-F_{\mathrm{r} 2} \sin \left(\alpha_{2}+\alpha_{1}\right)=0 \\
F_{\mathrm{w} 1} \sin \gamma-F_{\mathrm{w} 2} \sin \gamma-F_{\mathrm{r} 2} \cos \left(\alpha_{2}+\alpha_{1}\right)=0 \\
G \sin \beta-F_{\mathrm{a} 1}=0
\end{array},\right.
$$




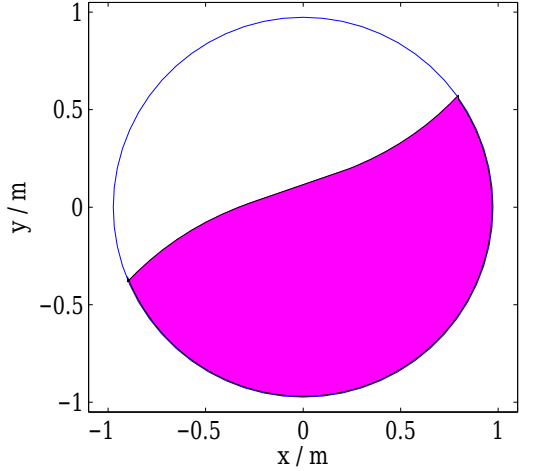

Figure 2. Shape of a concrete cross section at a certain time ( $\beta=$ $12^{\circ}, n=3 \mathrm{rmin}^{-1}$ ).

where $\alpha_{2}=\arccos \frac{e_{2}}{\sqrt{e_{1}^{2}+e_{2}^{2}}}$,

$\alpha_{1}=\arccos \frac{F_{\mathrm{c}}+e_{1} G \cos \beta / \sqrt{e_{1}^{2}+e_{2}^{2}}}{\left(G^{2} \cos ^{2} \beta+F_{\mathrm{c}}^{2}+2 e_{1} F_{\mathrm{c}} G \cos \beta / \sqrt{e_{1}^{2}+e_{2}^{2}}\right)^{1 / 2}}, e_{1}$ and $e_{2}$

are the vertical distance from gravity center to $x$ and $y$ axis. By solving Eq. (1), we can get the supporting forces $F_{\mathrm{a} 1}, F_{\mathrm{r} 1}$, $F_{\mathrm{r} 2}, F_{\mathrm{w} 1}$, and $F_{\mathrm{w} 2}$.

Since the concrete in the drum is a kind of slurry fluid, the distribution shape of the concrete changes with the rotation of the rotating drum. The shape of the concrete on a cross section should be as that shown in Fig. 2. Rotating speed can not be too high, otherwise, the concrete will form a ring distribution in the rotary drum, and can not form an effective mixing. However, the speed can not be too low, otherwise, most concrete will always be in a horizontal state and this can not achieve the mixing effect. Therefore, the speed should fall in a reasonable range.

Taking one particle concrete element as the object, the motion analysis is shown in Fig. 3. In the mixing process, the motion state of the concrete can be divided into two parts. One part of the concrete makes no movement in a circular area, the radius of which is $r_{1}$. Another part of the concrete undergoes a circular motion from point $\mathrm{A}$ to point $\mathrm{B}$, and then from point $\mathrm{B}$ along $\mathrm{BC}$ moving to $\mathrm{C}$ due to the effect of gravity, centrifugal force and friction force. And then repeat this process all the time ( $\mathrm{Li}, 2013)$.

The equation of dynamic mass center in the mixing process is deduced by literature ( $\mathrm{Li}, 2013)$ :

When $0 \leq t \leq \frac{\phi_{\max }-\phi_{0}}{\omega}$, the concrete moves in a circle, the dynamic mass center is:

$$
\left\{\begin{array}{l}
x_{1}=\frac{1}{S} \int_{r_{1}}^{r_{2}} \cos \phi \mathrm{d} \phi \mathrm{d} r \\
y_{1}=\frac{1}{S} \int_{r_{1}}^{r_{2}} \sin \phi \mathrm{d} \phi \mathrm{d} r
\end{array}\right.
$$

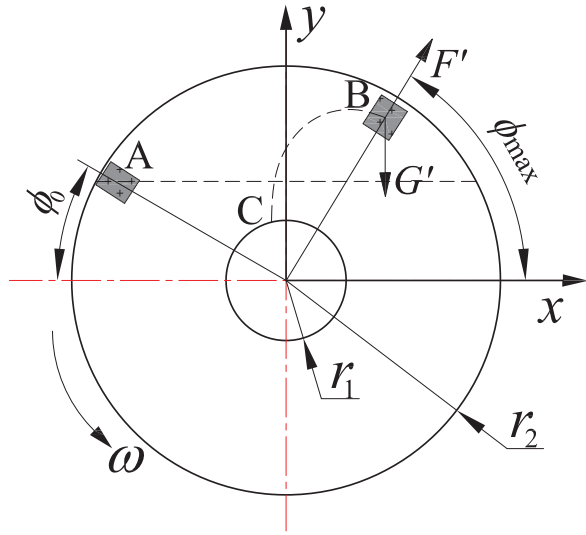

Figure 3. Schematic diagram of circumferential motion in concrete mixing process.

When $\frac{\phi_{\max }-\phi_{0}}{\omega}<t \leq \frac{2 \pi}{\omega}$, the concrete makes an oblique projectile motion, the dynamic mass center is:

$$
\left\{\begin{array}{l}
x_{2}=\frac{\int_{r_{1}}^{r_{2}} r \int_{\phi_{\mathrm{m}}}^{\phi}\left[r \cos \phi_{\max }-r\left(\phi-\phi_{\max }\right) \sin \phi_{\max }\right] \mathrm{d} \phi \mathrm{d} r}{S} \\
+\frac{\int_{r_{1}}^{r_{2}} r \int_{0}^{\phi_{\mathrm{m}}} \cos \phi \mathrm{d} \phi \mathrm{d} r}{S} \\
\frac{\int_{r_{1}}^{r_{2}} r \int_{\phi_{\mathrm{m}}}^{\phi}\left[r \sin \phi_{\max }-r\left(\phi-\phi_{\max }\right) \cos \phi_{\max }-0.5 \omega^{-2} g\left(\phi-\phi_{\max }\right)^{2}\right] \mathrm{d} \phi \mathrm{d} r}{S} \\
+\frac{\int_{r_{1}}^{r_{2}} r \int_{0}^{\phi_{\mathrm{m}}} \sin \phi \mathrm{d} \phi \mathrm{d} r}{S}
\end{array}\right.
$$

where $\phi_{\mathrm{m}}=\phi_{\max }-\phi_{0}, S$ is area of the particle concrete element. According to the Eqs. (2) and (3), the shape of concrete at any time any cross section can be obtained. On this basis, the mass center of each cross section can be calculated, and the eccentricities $e_{1}$ and $e_{2}$ can be confirmed. Finally, the centrifugal force $F_{\mathrm{c}}$ at different time will be obtained.

Based on the above theory, the distribution law of concrete in the mixing drum can be calculated numerically. Figure 4 shows the three dimensional distribution shape of concrete, where the inclination angle is $\beta=12^{\circ}$, and rotating speed is $n=3 \mathrm{rmin}^{-1}, 8 \mathrm{rmin}^{-1}, 15 \mathrm{rmin}^{-1}$. It can be found by comparing Fig. 4a, b, c that, for the same tilt angle, the concrete shapes are different under different rotating speeds. It can be seen from Fig. 4d that with the increase of the rotating speed, the highest level of concrete is increasing, the lowest liquid level is gradually reduced.

Figure 5 shows the influences of inclined angle on the concrete surface shape when $\beta=10^{\circ}, 12^{\circ}, 14^{\circ}, 16^{\circ}, n=$ $8 \mathrm{rmin}^{-1}$. When the speed is fixed, with the increase of the tilt angle, the concrete liquid level is gradually increasing, but the maximum value of liquid level is almost unchanged. Different cross sections have different concrete shapes. The 

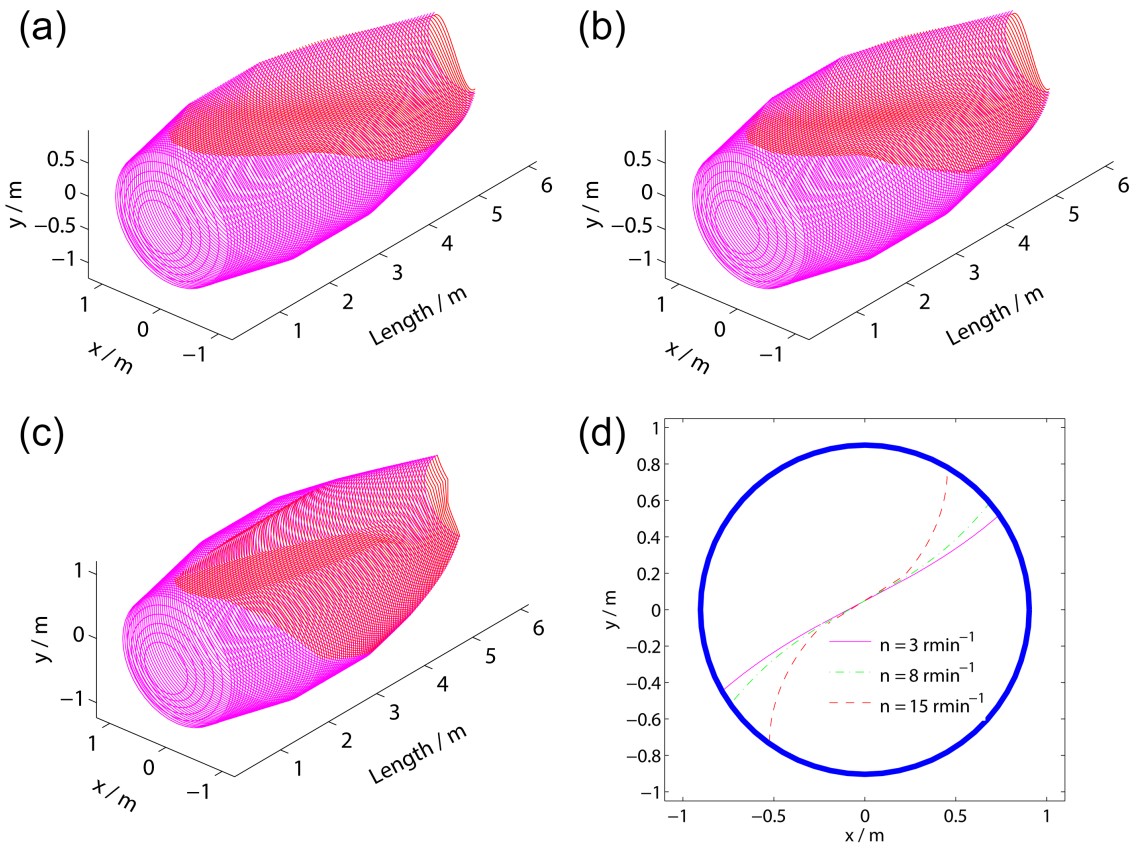

Figure 4. Shapes of the concrete with different speeds. (a) $\beta=12^{\circ}, n=3 \mathrm{rmin}^{-1}$. (b) $\beta=12^{\circ}, n=8 \mathrm{rmin}^{-1}$. (c) $\beta=12^{\circ}, n=15 \mathrm{rmin}{ }^{-1}$. (d) Concrete section shape $(z=4647 \mathrm{~mm})$.
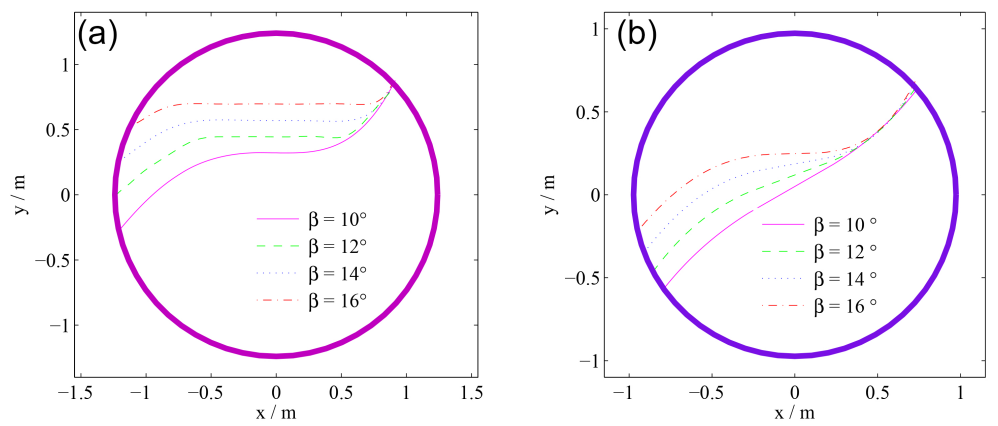

Figure 5. Shapes of the concrete with different tilt angles. (a) $z=3074 \mathrm{~mm}$. (b) $z=4647 \mathrm{~mm}$.

closer to the $z$ axis origin, the higher the concrete liquid level, and the more area of concrete is not moving with the mixing drum.

Figure $6 \mathrm{a}$ and $\mathrm{b}$ show the effect of the height of static concrete liquid level on concrete distribution shape when $\beta=12^{\circ}, n=8 \mathrm{rmin}^{-1}, h=0 \mathrm{~m},-0.10 \mathrm{~m},-0.15 \mathrm{~m}$, and $-0.20 \mathrm{~m}$ (at full load, the static height of the liquid level is as the benchmark $0 \mathrm{~m}$, lower than this is a negative value). It can be seen that with the decrease of static concrete liquid level height, the concrete surface level decreases at each cross section, however the maximum value of surface level is almost unchanged. Different cross sections have different concrete shapes. The position nearer to the $z$ axis origin will has smaller concrete liquid level value.

\section{Vibration model of mixing drum}

According to previous research, it can be known that the distribution shape of concrete in the drum is continuously changing when working, which will affect the vibration performance of the drum. This paper used transfer matrix method to analyze the dynamic performance of the mixing drum with considering the concrete shape changing.

The concrete mixing drum is lumped in a new system shown in Fig. 7. The system is a multiple degrees of freedom system consisting of $n$ rigid lumped-mass discs and $n-1$ mass-less elastic shaft segment. The supporting at both ends of the drum are simplified as spring-damping system. $F_{\mathrm{e} j}$ is the centrifugal force caused by the concrete. Through the mechanical analysis of the disc and shaft segment, the transfer matrix of each node is established at both ends of the 

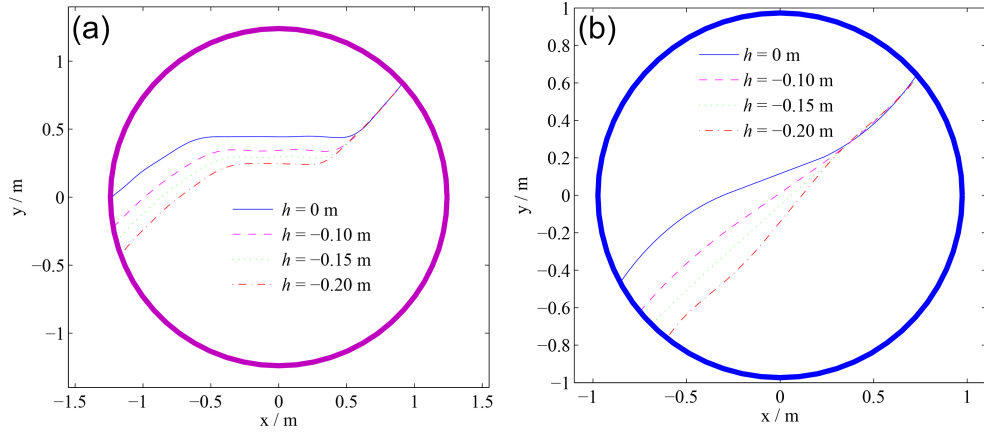

Figure 6. Shapes of the concrete with different concrete levels. (a) $z=3074 \mathrm{~mm}$. (b) $z=4647 \mathrm{~mm}$.

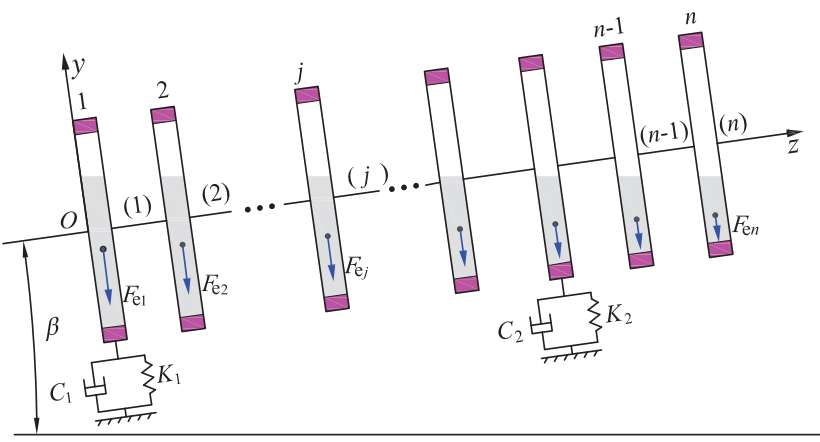

Figure 7. Lumped mass model of the concrete mixer rotating drum.

state vector. The equations consisted of transfer matrix can be solved by using the boundary conditions of the system, and the natural frequencies and the vibration modes of the system will be obtained.

Figure 8 is the dynamics analysis of shaft segment. The state vectors of both sides of the shaft segment can be expressed as

$Z_{j}=[y \theta M Q]_{j}^{\mathrm{T}}$,

where $y, \theta, M$, and $Q$ denote the transverse displacement, rotation angle, bending moment and shearing force on both sections. According to the mechanics theory (shearing deformation and higher modal responses are neglected here). A more representative approach has been stated in reference Matsubara et al. (1988), we have:

$$
\left\{\begin{array}{l}
Q_{j}^{\mathrm{R}}=Q_{j}^{\mathrm{L}} \\
M_{j}^{\mathrm{R}}=M_{j}^{\mathrm{L}}+Q_{j}^{\mathrm{L}} l_{j} \\
\theta_{j}^{\mathrm{R}}=\theta_{j}^{\mathrm{L}}+\frac{1}{(\mathrm{EI})_{j}} \int_{0}^{l_{j}} M_{j}^{\mathrm{R}} \mathrm{d} z=\theta_{j}^{\mathrm{L}}+\frac{M_{j}^{\mathrm{L}} l_{j}}{(\mathrm{EI})_{j}}+\frac{Q_{j}^{\mathrm{L}} l_{j}^{2}}{2(\mathrm{EI})_{j}} \\
y_{j}^{\mathrm{R}}=y_{j}^{\mathrm{L}}+\int_{0}^{l_{j}} \theta_{j}^{\mathrm{R}} \mathrm{d} z=y_{j}^{\mathrm{L}}+\theta_{j}^{\mathrm{L}} l_{j}+\frac{M_{j}^{\mathrm{L}} l_{j}^{2}}{2(\mathrm{EI})_{j}}+\frac{Q_{j}^{\mathrm{L}} l_{j}^{3}}{6(\mathrm{EI})_{j}}
\end{array}\right.
$$

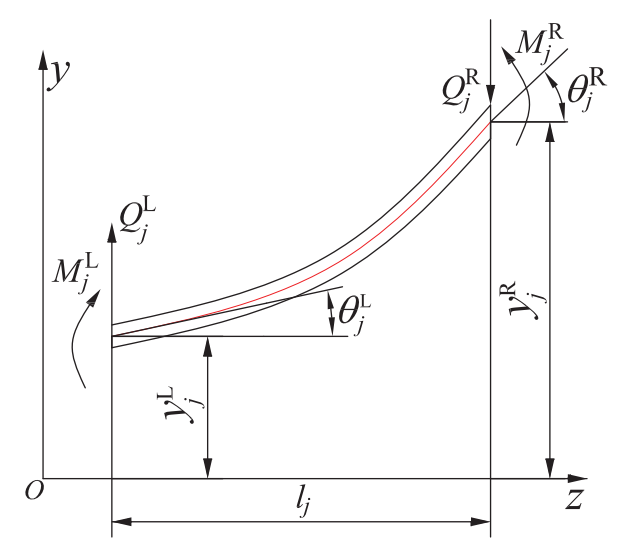

Figure 8. Mechanics analysis of shaft segment.

Equation (4) can be written in matrix form:

$$
\left[\begin{array}{c}
y \\
\theta \\
M \\
Q
\end{array}\right]_{j}^{\mathrm{R}}=\left[\begin{array}{cccc}
1 & l & l^{2} / 2 \mathrm{EI} & l^{3} / 6 \mathrm{EI} \\
0 & 1 & l / \mathrm{EI} & l^{2} / 2 \mathrm{EI} \\
0 & 0 & 1 & l \\
0 & 0 & 0 & 1
\end{array}\right]_{j}\left[\begin{array}{c}
y \\
\theta \\
M \\
Q
\end{array}\right]_{j}^{\mathrm{L}} .
$$

Reduce it to a simplified form:

$Z_{j}^{\mathrm{R}}=B_{j} Z_{j}^{\mathrm{L}}$,

where EI is the bending stiffness of the shaft segment. $l$ is the length of the shaft segment. The subscript $j$ indicates the shaft segment number. The superscript $\mathrm{L}$ and $\mathrm{R}$ represent the left-hand and right-hand respectively.

Figure 9 is the schematic diagram of mechanics analysis of tilt disk with concrete loading. On the basis of the force analysis, the relationship between the two sides of the cross section can be written as:

$\left[\begin{array}{c}y \\ \theta \\ M \\ Q\end{array}\right]_{j}^{\mathrm{R}}=\left[\begin{array}{cccc}1 & 0 & 0 & 0 \\ 0 & 1 & 0 & 0 \\ 0 & \left(J_{\mathrm{d}}-J_{\mathrm{p}}\right) \omega^{2} & 1 & 0 \\ m \omega^{2} & 0 & 0 & 1\end{array}\right]_{j}\left[\begin{array}{c}y \\ \theta \\ M \\ Q\end{array}\right]_{j}^{\mathrm{L}}+\left[\begin{array}{c}0 \\ 0 \\ -M_{\mathrm{a}} \\ -F_{\mathrm{e}}\end{array}\right]_{j}^{\mathrm{L}}$,

where $M_{\mathrm{a}}$ is the torque induced by the concrete, $F_{\mathrm{e}}$ is the centrifugal force induced in mixing process. $J_{\mathrm{d}}$ and $J_{\mathrm{p}}$ are the 


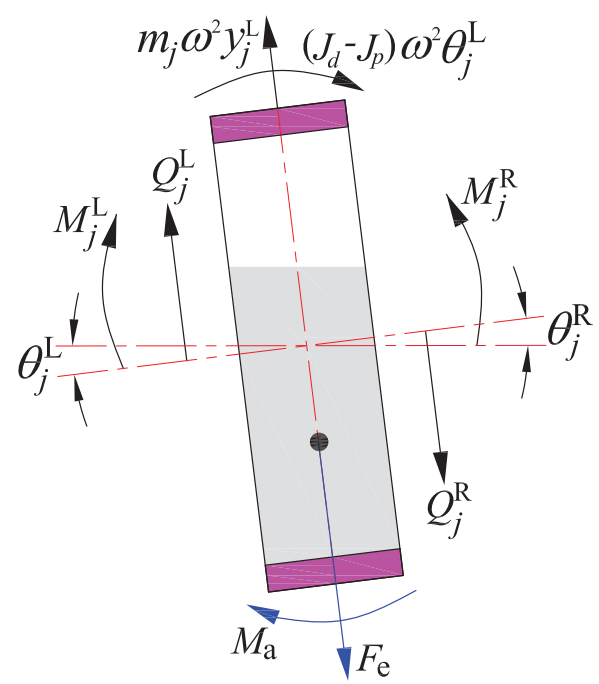

Figure 9. Mechanics analysis of tilt disk with concrete loading.

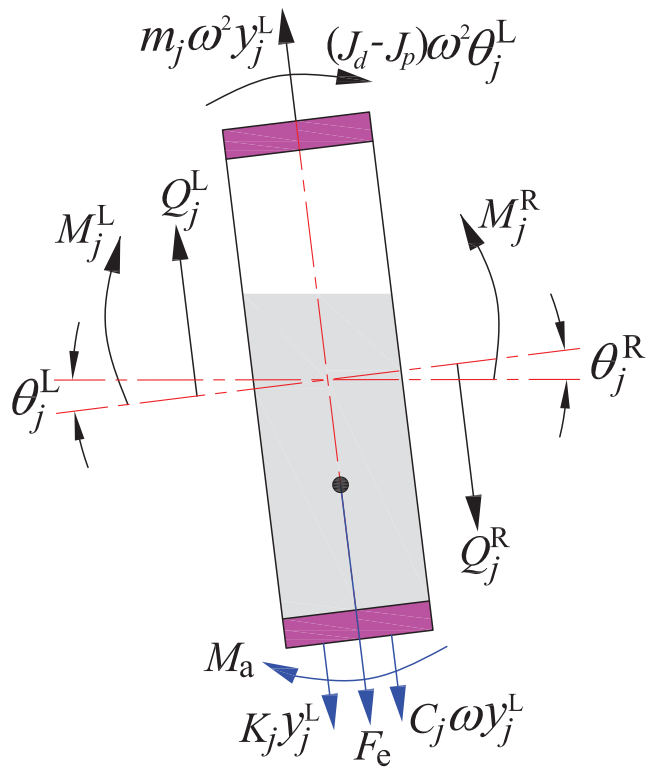

Figure 10. Mechanics analysis of tilt disk at supporting point.

diameter and polar moment of inertia of the disc respectively. $J_{\mathrm{p}}=m_{j}\left(R_{1 j}^{2}+R_{2 j}^{2}\right) / 2, J_{\mathrm{d}}=J_{\mathrm{p}} / 2 . R_{1}$ is the inner diameter of the tilt disk. $R_{2}$ is the outer diameter of the tilt disk.
Figure 10 shows the mechanics analysis of tilt disk at supporting point. The relationship between the two sides of the cross section can be expressed by matrix:

$$
\begin{aligned}
& {\left[\begin{array}{c}
y \\
\theta \\
M \\
Q
\end{array}\right]_{j}^{\mathrm{R}}=\left[\begin{array}{cccc}
1 & 0 & 0 & 0 \\
0 & 1 & 0 & 0 \\
0 & \left(J_{\mathrm{d}}-J_{\mathrm{p}}\right) \omega^{2} & 1 & 0 \\
m \omega^{2}-K-i C \omega & 0 & 0 & 1
\end{array}\right]_{j}\left[\begin{array}{c}
y \\
\theta \\
M \\
Q
\end{array}\right]_{j}^{\mathrm{L}}} \\
& +\left[\begin{array}{c}
0 \\
0 \\
-M_{\mathrm{a}} \\
-F_{\mathrm{e}}
\end{array}\right]_{j}^{\mathrm{L}}
\end{aligned}
$$

where $J_{\mathrm{d}}$ and $J_{\mathrm{p}}$ are the diameter and polar moment of inertia of the disc respectively. $\omega$ is the angular velocity, $m$ is the mass of the disk. $K$ and $C$ are the stiffness and damping at the supporting point.

Equations (7) and (8) can be simplified expression as:

As for the left supporting point, the transfer matrix between the two ends of the state vector can be calculated by using the Eq. (8) with $M_{\mathrm{a}}=0$ and $F_{\mathrm{e}}=0$.

$Z_{j}^{\mathrm{R}}=D_{j} Z_{j}^{\mathrm{L}}+F_{j}$

Through the Eqs. (6), (9a), the cross section state vector of the system can be expressed as:

$$
\left\{\begin{array}{l}
Z_{1}^{\mathrm{L}}=Z_{1}^{\mathrm{L}} \\
Z_{1}^{\mathrm{R}}=D_{1} Z_{1}^{\mathrm{L}}+F_{1} \\
Z_{2}^{\mathrm{L}}=B_{1} Z_{1}^{\mathrm{R}} \\
Z_{2}^{\mathrm{R}}=D_{2} Z_{2}^{\mathrm{L}}+F_{2} \\
\cdots \\
Z_{n}^{\mathrm{L}}=B_{n-1} Z_{n}^{\mathrm{R}} \\
Z_{n}^{\mathrm{R}}=D_{n} Z_{n}^{\mathrm{L}}+F_{n}
\end{array}\right.
$$

Though the recursive relation of Eq. (10), we can establish the relation between $Z_{n}^{\mathrm{R}}$ and $Z_{1}^{\mathrm{L}}$ :

$$
\left[\begin{array}{c}
y \\
\theta \\
M \\
Q
\end{array}\right]_{n}^{\mathrm{R}}=\left[\begin{array}{llll}
a_{11} & a_{12} & a_{13} & a_{14} \\
a_{21} & a_{22} & a_{23} & a_{24} \\
a_{31} & a_{32} & a_{33} & a_{34} \\
a_{41} & a_{42} & a_{43} & a_{44}
\end{array}\right]_{n}\left[\begin{array}{c}
y \\
\theta \\
M \\
Q
\end{array}\right]_{1}^{\mathrm{L}}+\left[\begin{array}{l}
b_{1} \\
b_{2} \\
b_{3} \\
b_{4}
\end{array}\right]_{n-1}
$$

Bring the boundary conditions $M_{1}^{\mathrm{L}}=0, Q_{1}^{\mathrm{L}}=0, M_{n}^{\mathrm{R}}=0$, $Q_{n}^{\mathrm{R}}=0$ into transfer matrix (11), we can get:

$$
\left[\begin{array}{c}
M \\
Q
\end{array}\right]_{n}^{\mathrm{R}}=\left[\begin{array}{ll}
a_{31} & a_{32} \\
a_{41} & a_{42}
\end{array}\right]\left[\begin{array}{l}
y \\
\theta
\end{array}\right]_{1}^{\mathrm{L}}+\left[\begin{array}{l}
b_{3} \\
b_{4}
\end{array}\right]_{n-1}=\left[\begin{array}{l}
0 \\
0
\end{array}\right]
$$

By solving Eq. (12), the unbalanced response of the mixing drum can be obtained. 


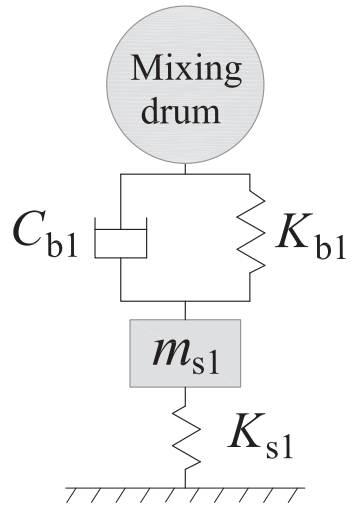

Figure 11. Stiffness and damping model at left supporting point.

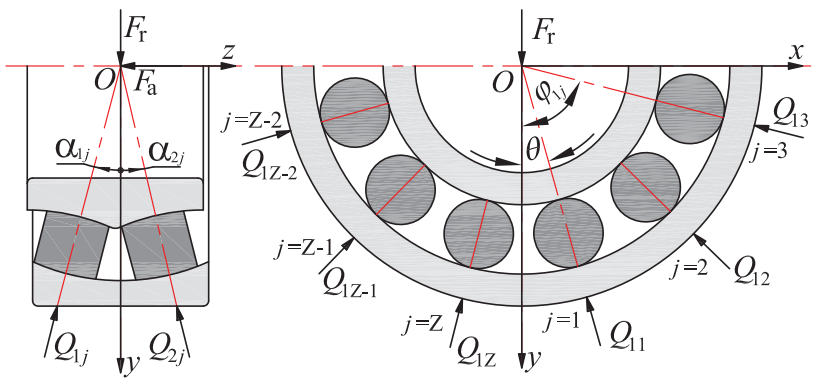

Figure 12. Mechanics analysis of spherical roller bearing.

\section{Stiffness and damping}

\subsection{Stiffness and damping at left supporting point}

The stiffness and damping at left supporting point is composed of the stiffness and damping of the gear reducer's main bearing and the front bracket. The stiffness and damping model is shown in Fig. 11. The mass of the bearing and the damping of the front bracket are very small, and can be neglected. Thus, the spherical roller bearing (SRB) in the gear reducer is equivalent to a spring-damping system, and the front bracket is equivalent to a spring-mass system. Both simplified models are in series. According to the series-parallel calculation formula of stiffness and damping, the total stiffness and damping can be expressed as (Wen et al. 1999):

$K_{\mathrm{left}}=\frac{K_{\mathrm{b} 1}\left(K_{\mathrm{s} 1}-m_{\mathrm{s} 1} \omega^{2}\right) / \cos ^{2} \beta}{K_{\mathrm{b} 1}+\left(K_{\mathrm{s} 1}-m_{\mathrm{s} 1} \omega^{2}\right) / \cos ^{2} \beta}$,

$C_{\text {left }}=C_{\mathrm{b} 1}$,

where $K_{\mathrm{b} 1}$ and $C_{\mathrm{b} 1}$ are the stiffness and damping of SRB, $K_{\mathrm{s} 1}$ and $m_{\mathrm{s} 1}$ are the vertical stiffness and mass of front bracket.

The stiffness and damping calculation method of double row spherical roller bearing (as shown in Fig. 12) has not been studied in detail at present. However, based on the definition of stiffness, the contact stiffness of the $j$ th roller-raceway in the first and second row of the bearing is (Houpert, 2001):

$$
\begin{gathered}
K_{\mathrm{SZ} \__{-} 1 j}=\lim _{\substack{V Q_{1 j} \rightarrow 0 \\
V \delta_{1 j} \rightarrow 0}} \frac{V Q_{1 j}}{V \delta_{1 j}} \\
=\frac{0.8752 E^{\prime 2 / 3} Q_{1 j}^{1 / 3}}{\left(R_{x \mathrm{i}}^{-0.0590}+R_{x \mathrm{o}}^{-0.0590}\right)\left(R_{y \mathrm{i}}^{-0.2743}+R_{y \mathrm{o}}^{-0.2743}\right)} \\
K_{\mathrm{SZ} \_2 j}=\lim _{V Q_{1 j} \rightarrow 0} \frac{V Q_{2 j}}{V \delta_{2 j}} \\
=\frac{V \delta_{1 j} \rightarrow 0}{\left(R_{x \mathrm{i}}^{-0.0590}+R_{x \mathrm{o}}^{-0.0590}\right)\left(R_{y \mathrm{i}}^{-0.2743}+R_{y 0}^{-0.2743}\right)} \\
\left\{\begin{array}{l}
R_{x \mathrm{i}}=\frac{1}{\rho_{\mathrm{I}}+\rho_{\mathrm{Ii}}} \\
R_{y \mathrm{i}}=\frac{1}{\rho_{\mathrm{II}}+\rho_{\mathrm{IIi}}} \\
R_{x \mathrm{o}}=\frac{1}{\rho_{\mathrm{I}}+\rho_{\mathrm{Io}}} \\
R_{y \mathrm{o}}=\frac{1}{\rho_{\mathrm{II}}+\rho_{\mathrm{IIo}}}
\end{array}\right.
\end{gathered}
$$

where $Q_{1 j}$ and $Q_{2 j}$ are normal loads acting on the two rows of rollers, and can be obtained according to the method of literature (Ma et al., 2016). $E^{\prime}\left(\mathrm{Nm}^{-2}\right)$ is the equivalent elastic modulus. $R_{x}$ is the equivalent radius of the contact point along rolling direction of the roller. $R_{y}$ is the equivalent radius of the contact point perpendicular to the rolling direction of the roller. The principal radii of spherical roller of contact in $x y$ and $x z$ planes are $\rho_{\mathrm{I}}=\frac{2}{D}, \rho_{\mathrm{II}}=\frac{1}{R}$. The principal radii of the inner race of contact in $x y$ and $x z$ planes are $\rho_{\mathrm{Ii}}=\frac{2 \gamma}{D(1-\gamma)}, \rho_{\mathrm{IIi}}=-\frac{1}{r_{\mathrm{i}}}$. The principal radii of the outer race of contact in $x y$ and $x z$ planes are $\rho_{\text {Io }}=-\frac{2 \gamma}{D(1+\gamma)}$, $\rho_{\text {IIo }}=-\frac{1}{r_{0}} . D$ is the roller diameter. $R$ is the roller contour radius. $r_{\mathrm{i}}$ is the inner contour radius. $r_{\mathrm{O}}$ is the outer contour radius. $\gamma=D \cdot \cos \alpha_{\mathrm{c}} / d_{\mathrm{m}} \cdot \alpha_{\mathrm{c}}$ is the contact angle. $d_{\mathrm{m}}$ is the pitch circle diameter.

The oil film stiffness of the first and second column of roller $j$ is (Yang et al., 2016):

$$
\begin{gathered}
K_{\text {oil_1j }}=\lim _{\substack{\Delta Q_{1 j} \rightarrow 0 \\
\Delta h_{\min } \rightarrow 0}} \frac{\Delta Q_{1 j}}{\Delta h_{\min }}=\frac{Q_{1 j}^{1.073}}{0.073 \lambda} \\
K_{\text {oil_2j }}=\lim _{\substack{\Delta Q_{2 j} \rightarrow 0 \\
\Delta h_{\min } \rightarrow 0}} \frac{\Delta Q_{2 j}}{\Delta h_{\min }}=\frac{Q_{2 j}^{1.073}}{0.073 \lambda} \\
\lambda=3.63 E^{\prime-0.117} \alpha^{0.49}\left(\eta_{0} v_{\mathrm{m}}\right)^{0.68}\left[\left(1-e^{-0.68 k_{\mathrm{i}}}\right) R_{x \mathrm{i}}^{0.466}\right. \\
\left.+\left(1-e^{-0.68 k_{\mathrm{o}}}\right) R_{x \mathrm{o}}^{0.466}\right]
\end{gathered}
$$


The comprehensive radial stiffness of SRB is:

$$
\begin{gathered}
K_{\mathrm{b} 1}=\sum_{j=1}^{j=Z}\left(\left(K_{\mathrm{sz} \_1 j}^{-1}+K_{\mathrm{oil} \_1 j}^{-1}\right)^{-1} \cos \varphi_{1 j} \cos \alpha_{1 j}\right. \\
\left.+\left(K_{\mathrm{sz} \_2 j}^{-1}+K_{\text {oil_ } 2 j}^{-1}\right)^{-1} \cos \varphi_{2 j} \cos \alpha_{2 j}\right),
\end{gathered}
$$

where $\alpha\left(\mathrm{m}^{2} \mathrm{~N}^{-1}\right)$ is the pressure-viscosity coefficient of the lubricant. $\eta_{0}(\mathrm{Pas})$ is the dynamic viscosity of lubricant under the pressure $0.1 \mathrm{MPa} . v_{\mathrm{m}}\left(\mathrm{m} \mathrm{s}^{-1}\right)$ is the average speed of rolling bearings. $e$ is the base of natural logarithms $(e=2.71828) . k$ is the ellipticity ratio. $\varphi_{1 j}$ and $\varphi_{2 j}$ are the azimuth of the rollers. $\alpha_{1 j}$ and $\alpha_{2 j}$ are the actual contact angles of roller-raceway contact. $Z$ is the number of single row rollers for SRB. Subscript i and o denote the inner and outer rings of the bearing.

According to the definition of damping $C=\lim _{\Delta \rightarrow 0} \frac{\Delta Q}{\Delta u_{z}}$, the damping in the first row of the bearing's roller $j$ between inner raceway and outer raceway can be obtained (Yang et al., 2016; Hamrock and Dowson, 1981):

$$
\left\{\begin{array}{l}
C_{1 j_{-} i}=\frac{2.16 a R_{x \mathrm{i}}^{0.831} Q_{1 j}^{0.1095}}{E^{\prime-0.1755} \alpha^{0.735} \eta_{0}^{0.02} v_{\mathrm{m}}^{1.02}\left(1-e^{-0.68 k_{\mathrm{i}}}\right)^{1.5}} \\
C_{1 j_{-} o}=\frac{2.16 a R_{x \mathrm{o}}^{0.831} Q_{1 j}^{0.1095}}{E^{\prime-0.1755} \alpha^{0.735} \eta_{0}^{0.02} v_{\mathrm{m}}^{1.02}\left(1-e^{-0.68 k_{\mathrm{o}}}\right)^{1.5}}
\end{array} .\right.
$$

According to damping series relation of bearing, the comprehensive damping in first row of the bearing at roller $j$ is:

$C_{1 j}=\left(C_{1 j_{-} \mathrm{i}}^{-1}+C_{1 j_{-} \mathrm{o}}^{-1}\right)^{-1} \cos \varphi_{1 j} \cos \alpha_{1 j}$.

Also, the comprehensive damping in the second row of the bearing at roller $j$ is:

$C_{2 j}=\left(C_{2 j_{-} \mathrm{i}}^{-1}+C_{2 j_{-} \mathrm{O}}^{-1}\right)^{-1} \cos \varphi_{2 j} \cos \alpha_{2 j}$.

The comprehensive damping of SRB is:

$C_{\mathrm{b} 1}=\sum_{j=1}^{j=Z}\left(C_{1 j}+C_{2 j}\right)$.

\subsection{Stiffness and damping at right supporting point}

The stiffness and damping at right supporting point consists of the stiffness and damping of the wheel-drum, bearing in the wheel and the rear bracket. Fig.13 is the stiffness and damping model of a single rolling wheel. According to the series-parallel calculation formula of stiffness and damping, the total stiffness and damping can be expressed as (Wen, et al., 1999):

$K_{\text {right }}=\frac{K_{\mathrm{bw}}\left(K_{\mathrm{s} 2} / \cos ^{2} \beta-m_{\mathrm{s} 2} \omega^{2}\right) \cos ^{2} \gamma}{K_{\mathrm{bw}}+K_{\mathrm{s} 2} / \cos ^{2} \beta-m_{\mathrm{s} 2} \omega^{2}}$,

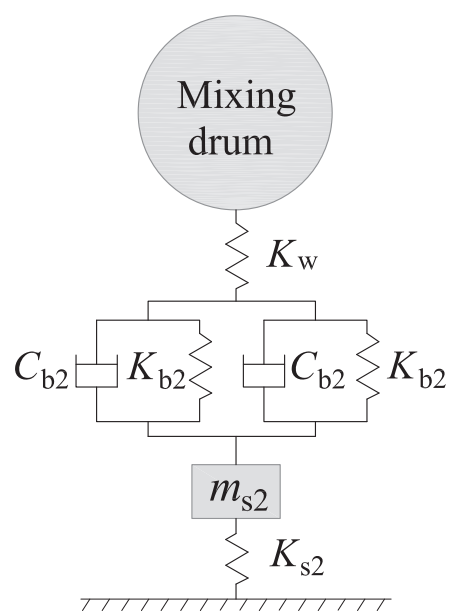

Figure 13. Stiffness and damping model of a single rolling wheel.

Table 1. Structural parameters of concrete mixer rotating drum.

\begin{tabular}{lr}
\hline Parameters & Value \\
\hline Length of front cone $L_{1}(\mathrm{~mm})$ & 1550 \\
Length of cylindrical section $L_{2}(\mathrm{~mm})$ & 1400 \\
Length of middle cone $L_{3}(\mathrm{~mm})$ & 1050 \\
Length of posterior cone $L_{4}(\mathrm{~mm})$ & 2200 \\
Length from wheel to bottom $L_{5}(\mathrm{~mm})$ & 890 \\
Total length of the drum $L(\mathrm{~mm})$ & 6490 \\
Diameter of drum head $D_{1}(\mathrm{~mm})$ & 1933 \\
Diameter of cylindrical drum $D_{2}(\mathrm{~mm})$ & 2480 \\
Diameter of middle drum $D_{3}(\mathrm{~mm})$ & 2110 \\
Diameter of raceway $D_{4}(\mathrm{~mm})$ & 1642 \\
Diameter of posterior drum $D_{5}(\mathrm{~mm})$ & 1132 \\
Diameter of drum entrance $D_{6}(\mathrm{~mm})$ & 560 \\
\hline
\end{tabular}

$C_{\text {rightt }}=2\left(C_{\mathrm{b} 21}+C_{\mathrm{b} 22}\right) \cos \gamma$,

where

$K_{\mathrm{bw}}=\left[\left(\frac{1}{2 K_{\mathrm{b} 21}}+\frac{1}{K_{\mathrm{w} 1}}\right)^{-1}+\left(\frac{1}{2 K_{\mathrm{b} 22}}+\frac{1}{K_{\mathrm{w} 2}}\right)^{-1}\right]^{-1} \cos ^{2} \gamma$,

$K_{\mathrm{b} 21}, C_{\mathrm{b} 21}$ and $K_{\mathrm{b} 22}, C_{\mathrm{b} 22}$ are the stiffness and damping of the bearings in first roller wheel and the second roller wheel. Literature ( $\mathrm{Li}$ et al., 2016) gives the calculation method of radial stiffness $K_{\mathrm{w} 1}, K_{\mathrm{w} 2}$ between roller wheel and rotary drum. Literature (Wu, 2011) lists the parameters $K_{\mathrm{s} 2}, m_{\mathrm{s} 2}$.

\section{Specific example analysis}

The dynamic performance of a concrete mixing truck's mixing drum produced by a company is studied in this paper. The structure of the mixing drum is shown in Fig. 1, and the parameters of the drum are listed in Table 1. The main parameters of the gear reducer's main bearing are shown in Table 2. The type of the tapered roller bearing supporting the roller wheel is 32311 . 


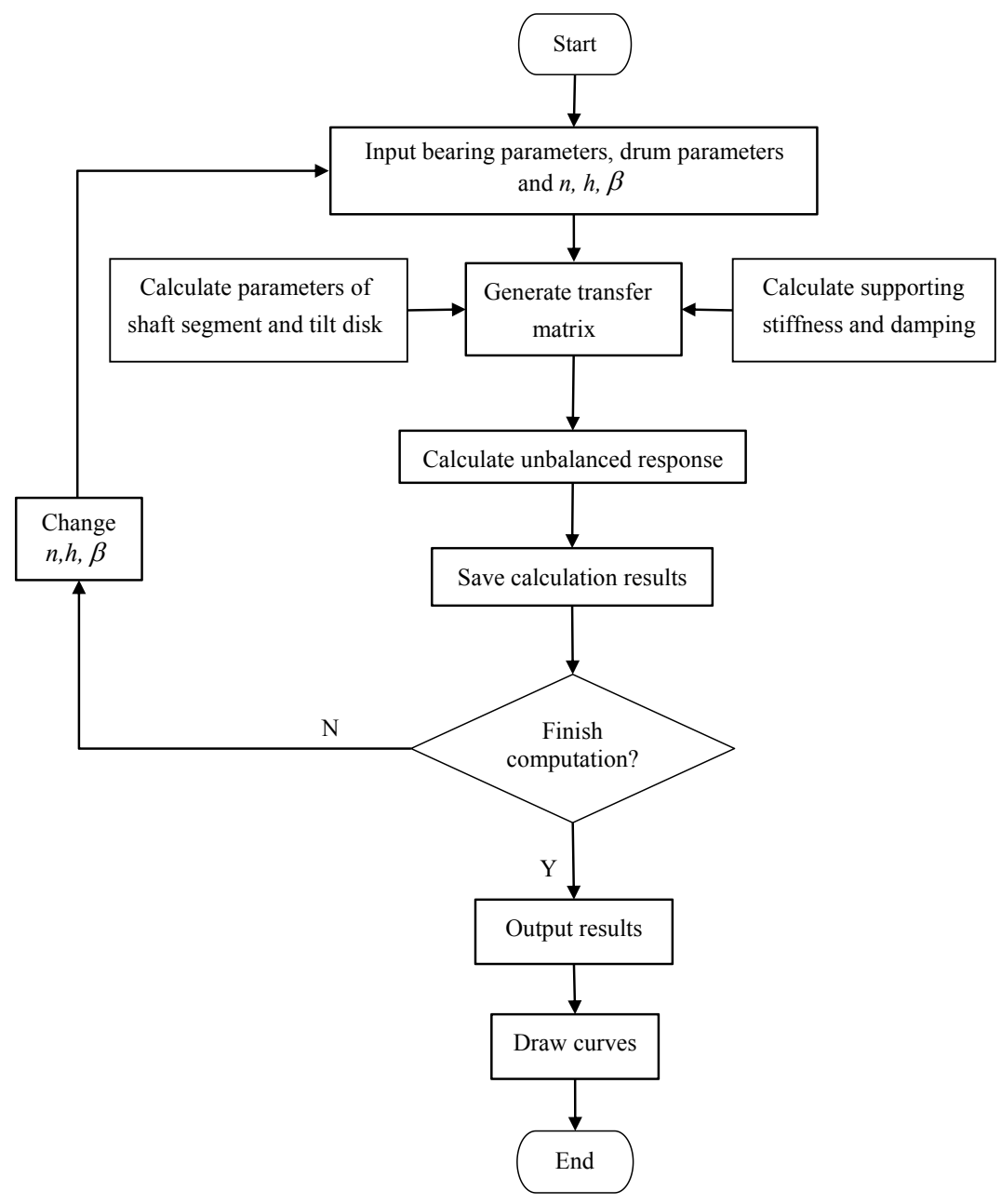

Figure 14. Calculation flow chart.
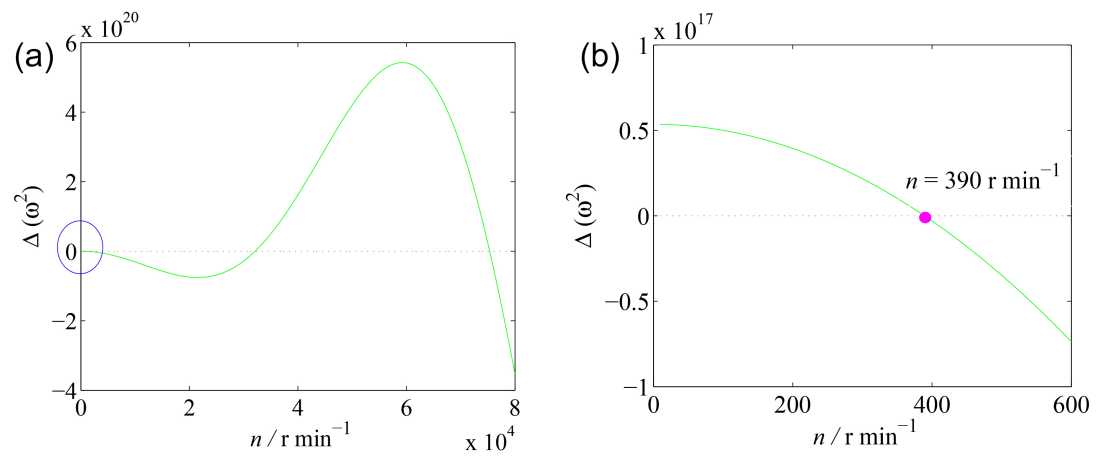

Figure 15. Residual volume $\Delta\left(\omega^{2}\right)$ curve. (a) Residual volume curve $n-\Delta\left(\omega^{2}\right)$. (b) Local enlarged drawing of (a).

By using the calculation model established in this paper, a series of curves are obtained by Matlab programming. Figure 14 is the calculation flow chart of the programming.

Figure $15 \mathrm{a}$ is the residual volume curve when calculating the critical speed of the mixing drum. Figure $15 \mathrm{~b}$ is local enlarged drawing of Fig. 15a. The critical speed of the drum is at the intersection point of the curve and $y=0$. It can be seen from Fig. 15b, first-order critical speed of the drum is $390 \mathrm{r} \mathrm{min}^{-1}$. In the actual working process, the rotating speed of the mixing drum can not reach the critical speed. Therefore, this paper only study the dynamic performance of the drum within $n=3 \sim 15 \mathrm{rmin}^{-1}$. 

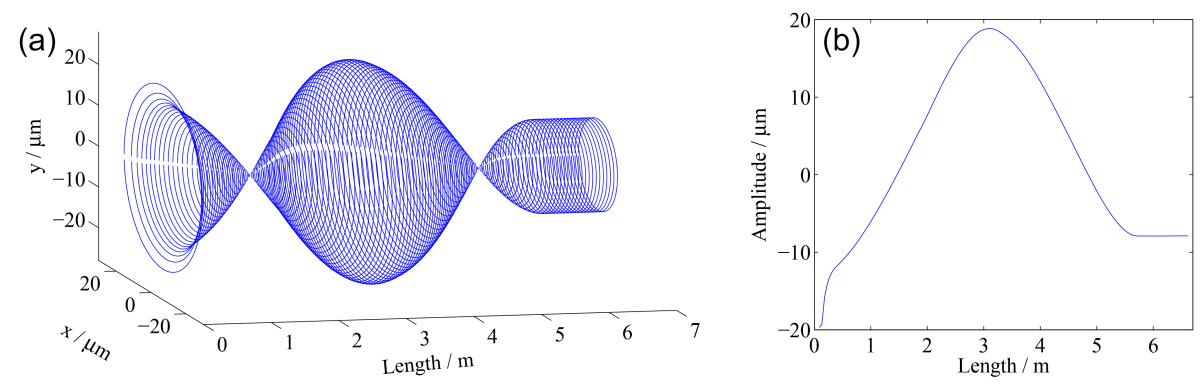

Figure 16. Vibration amplitude of the concrete mixing drum. (a) Vibration mode. (b) Response curve.

Table 2. Main parameters of the reducer bearing.

\begin{tabular}{lr}
\hline Parameters & Value \\
\hline Inner contour radius (mm) & 98.85 \\
Outer contour radius (mm) & 98.85 \\
Roller diameter (mm) & 24 \\
Roller contour radius (mm) & 97.71 \\
Number of single row roller & 19 \\
Bearing inside diameter (mm) & 120 \\
Bearing outside diameter $(\mathrm{mm})$ & 215 \\
Bearing width $(\mathrm{mm})$ & 80 \\
Radial clearance $(\mathrm{mm})$ & 0.083 \\
Nominal contact angle $\left({ }^{\circ}\right)$ & 10 \\
Elastic modulus $\left(\mathrm{Nm}{ }^{-2}\right)$ & $2.06 \times 10^{11}$ \\
Poisson's ratio & 0.3 \\
Dynamic viscosity of lubricant $\left(\mathrm{Pas}^{2}\right)$ & 0.1362 \\
Pressure-viscosity coefficient $\left(\mathrm{m}^{2} \mathrm{~N}^{-1}\right)$ & $2.03 \times 10^{-8}$
\end{tabular}

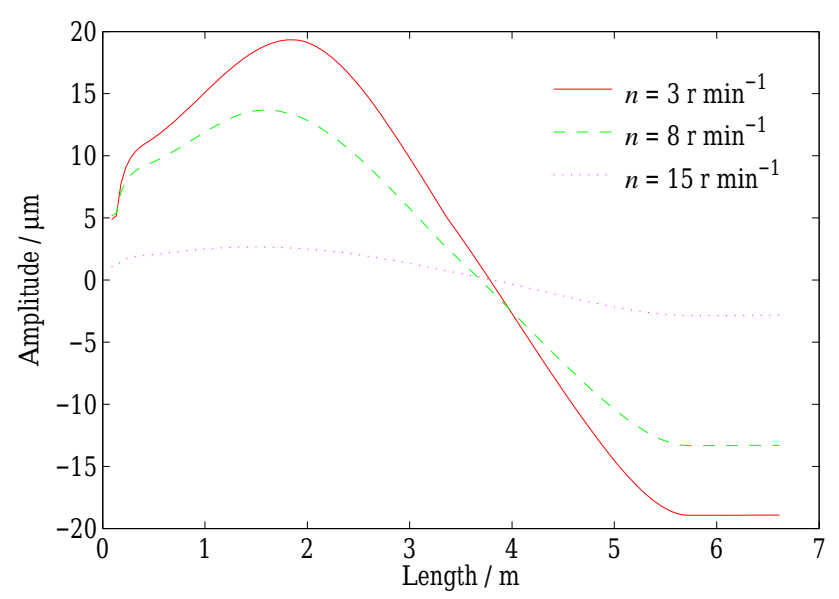

Figure 17. Effect of rotation speed on drum's vibration amplitude.

Figure $16 \mathrm{a}$ and $\mathrm{b}$ show vibration curve of the mixing drum with $n=3 \mathrm{rmin}^{-1}, \beta=16^{\circ}, h=0 \mathrm{~m}$. As shown in Fig. $16 \mathrm{~b}$, the response curve distributes asymmetrically. The vibration amplitude first increases to the maximum value and then gradually decrease $(L=5.6 \mathrm{~m})$. The vibration amplitude of the drum is almost not changed at the right side of the roller

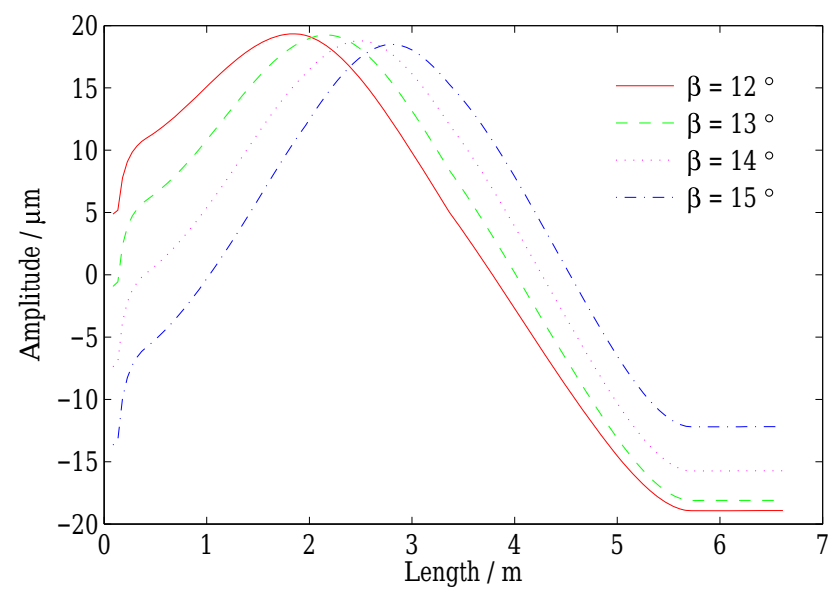

Figure 18. Effect of the drum's tilt angle on vibration amplitude.

wheel supporting point. This vibration mode is related to the distribution of the concrete in the drum.

Figure 17 depicts the influences of rotating speed on the dynamic properties of the mixing drum when $\beta=12^{\circ}$ and $h=0 \mathrm{~m}$. The vibration amplitude of the mixing drum decreases with the increase of the rotating speed. This is due to the wider distribution of the concrete along the rotating direction and the more obviously symmetrical distribution of concrete which result in the great decrease of concrete's eccentricity. Although the rotating speed increases, the exciting force becomes smaller due to the great decrease of concrete's eccentricity.

Figure 18 shows the effects of drum's inclination angle on the vibration properties when $n=3 \mathrm{rmin}^{-1}$ and $h=0$. The peak amplitude gradually moves to the right with the inclination angle increasing. The vibration amplitude value of the peak's left side decreases when tilt angle increases, while the right side increases. The peak amplitude is almost unchanged and gradually moves to the right with the inclination angle increasing, which is caused by the right movement along the axial direction of the maximum eccentric position of the concrete in the mixing drum with the increase of the inclination angle. 


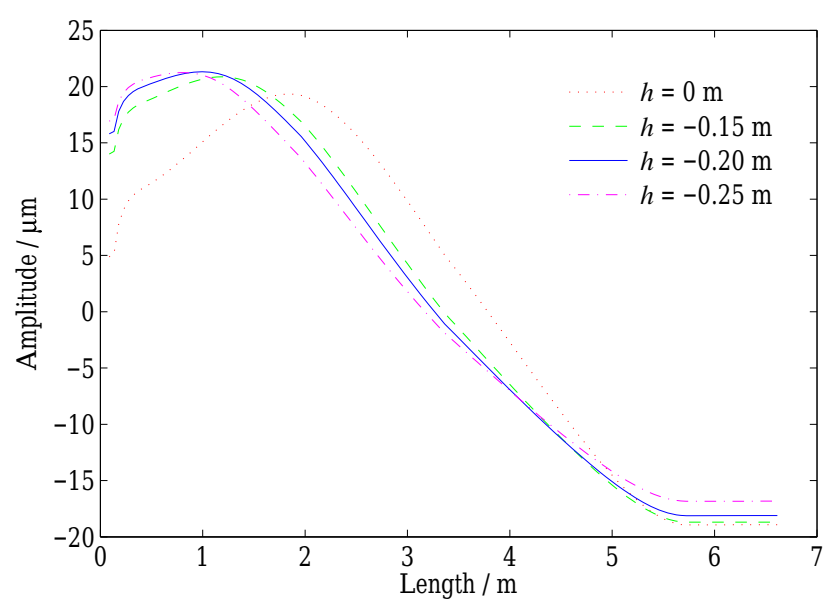

Figure 19. Effect of concrete level on the rotary drum's vibration amplitude.

Figure 19 presents the influences of concrete's liquid level height when $n=3 \mathrm{rmin}^{-1}$ and $\beta=12^{\circ}$. The maximum unbalanced response amplitude of the drum increases with the decrease of concrete liquid level height $h$, which is because that when $h$ decreases, the eccentric distance of the concrete in the mixing drum is gradually increased which result in the increase of concrete's maximum centrifugal force. It can also be seen that the vibration peak amplitude gradually moves to the left with the decrease of $h$.

\section{Conclusions}

A mathematical formulation has been derived through the force analysis for calculating the supporting force. The calculation method of the concrete distribution shape in the rotary drum is developed based on some others' previous studies. The effects of rotating speed, tilting angle and concrete liquid level on concrete shape are analyzed. A new transfer matrix is built with considering the concrete geometric distribution shape. The stiffness and damping calculation models of spherical roller bearings and rolling wheels are established. On the basis of these analysis, a method for calculating the dynamic performance of the mixing drum is established.

The effects of rotating speed, inclination angle and concrete liquid level on the vibration performance of the mixing drum are studied with a specific example. Speed-amplitude curves, inclination angle-amplitude curves and concrete liquid level height-amplitude curves are obtained. It is found that the vibration mode curves of the drum distribute asymmetrically. And with the increase of rotating speed, the vibration amplitude of the mixing drum decreases, the peak amplitude gradually moves to the right with the inclination angle increasing, the amplitude value of the peak's left side decreases when tilt angle increases, while the right side increases. The maximum unbalanced response amplitude of the drum increases with the decrease of concrete liquid level height, and the vibration peak moves to the left.

Data availability. All the data used in this manuscript can be obtained by requesting from the corresponding author. 


\section{Appendix A: Notations}

$a_{0} \quad$ distance between mass center and left supporting $(\mathrm{mm})$

C damping $\left(\mathrm{Ns} \mathrm{m}^{-1}\right)$

$e \quad$ eccentricity (mm)

E Young modulus (MPa)

$E^{\prime} \quad$ equivalent Young's modulus $(\mathrm{MPa})$

$F_{\text {a }} \quad$ axial force $(\mathrm{N})$

$F_{\mathrm{c}} \quad$ centrifugal force $(\mathrm{N})$

$F_{\mathrm{r}} \quad$ radial force $(\mathrm{N})$

$F_{\mathrm{W}} \quad$ roller wheel supporting force (N)

$G \quad$ gravity (N)

$J_{\mathrm{d}} \quad$ diameter moment of inertia of the disc

$J_{\mathrm{p}} \quad$ polar moment of inertia of the disc

$k$ ratio of equivalent radii of roller-raceway contact

$K \quad$ stiffness $\left(\mathrm{Nm}^{-1}\right)$

$L \quad$ drum length ( $\mathrm{mm})$

$m \quad$ disc mass $(\mathrm{kg})$

$M \quad$ bending moment $(\mathrm{Nm})$

$M_{\mathrm{a}} \quad$ additional bending moment by gravity $(\mathrm{Nm})$

$n \quad$ rotating speed $\left(\mathrm{rmin}^{-1}\right)$

$Q \quad$ shearing force (N)

$R_{x} \quad$ equivalent radius of roller-inner raceway

contact in rolling direction $(\mathrm{mm})$

$R_{y} \quad$ equivalent radius of roller-inner raceway contact

perpendicular to the rolling direction $(\mathrm{mm})$

$v_{m} \quad$ average speed of bearing $\left(\mathrm{m} \mathrm{s}^{-1}\right)$

$Z \quad$ roller's number in each row of SRB

$\alpha \quad$ pressure-viscosity coefficient of lubricant $\left(\mathrm{m}^{2} \mathrm{~N}^{-1}\right)$

$\beta \quad$ inclination angle $\left(^{\circ}\right)$

$\gamma \quad$ angle between roller wheel center

and drum center $\left(^{\circ}\right)$

$\delta \quad$ normal elastic deformation of roller-raceway contact (mm)

$\eta_{0} \quad$ dynamic viscosity of lubricant (Pas)

$\theta \quad$ rotation angle of the section ( $\mathrm{rad})$

$\varphi \quad$ azimuth angle of roller ( $\mathrm{rad})$

$\omega \quad$ angular velocity $\left(\mathrm{rads}^{-1}\right)$

Superscript

L left-hand

$\mathrm{R} \quad$ right-hand

Subscript

a axial direction

inner raceway

serial number of lumped disc

$1 j \quad$ serial number of roller in first row of SRB

$2 j \quad$ serial number of roller in second row of SRB

left left supporting point

o outer raceway

$r \quad$ radial direction

right right supporting point

$x \quad$ along the coordinate direction $x$

$y \quad$ along the coordinate direction $y$

$z \quad$ along the coordinate direction $z$

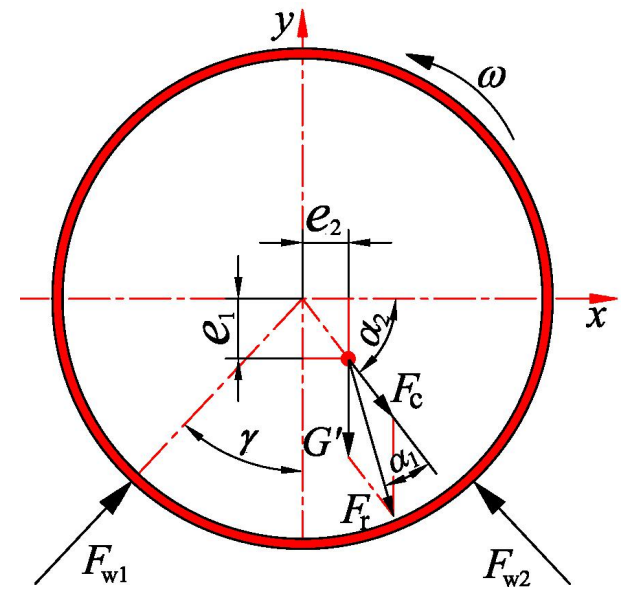

Figure B1. Force analysis of the mixing drum.

\section{Appendix B: Force analysis of the mixing drum}

The force analysis of the mixing is shown in Figs. 1 and B1.

According to the force balance principle, the forces of the mixing drum in radial direction are balanced:

$F_{\mathrm{r} 1}+F_{\mathrm{r} 2}=F_{\mathrm{r}}$,

$F_{\mathrm{r}}$ is the resultant force of $F_{\mathrm{c}}$ and $G^{\prime}$, the value of which is:

$F_{\mathrm{r}}=\left(G^{\prime 2}+F_{\mathrm{c}}^{2}+2 e_{1} G^{\prime} F_{\mathrm{c}} / \sqrt{e_{1}^{2}+e_{2}^{2}}\right)^{\frac{1}{2}}$,

where $G^{\prime}$ is a component of $G$, and it's direction is perpendicular to the axis:

$G^{\prime}=G \cos \beta$.

Combine Eqs. (A1), (A2) and (A3) into an equation, we can get:

$F_{\mathrm{r} 1}+F_{\mathrm{r} 2}-\left(G^{2} \cos ^{2} \beta+F_{\mathrm{c}}^{2}+2 e_{1} G F_{\mathrm{c}} \cos \beta / \sqrt{e_{1}^{2}+e_{2}^{2}}\right)^{1 / 2}=0$.

The forces of the mixing drum in axial direction are balanced:

$F_{\mathrm{a} 1}-G \sin \beta=0$.

According to the moment balance principle, the moment at the mass center is balanced:

$a_{0} F_{\mathrm{r} 1}+G \sin \beta \sqrt{e_{1}^{2}+e_{2}^{2}}-F_{\mathrm{r} 2}\left(L_{6}-L_{5}-a_{0}\right)=0$.

$F_{\mathrm{r} 2}$ and $F_{\mathrm{r}}$ have the opposite direction, and $F_{\mathrm{r} 2}$ is the resultant force of $F_{\mathrm{w} 1}$ and $F_{\mathrm{w} 2}$, which means:

$F_{\mathrm{r} 2}=F_{\mathrm{w} 1}+F_{\mathrm{w} 2}$. 
Equation (A4) can be written as:

$F_{\mathrm{w} 1} \cos \gamma+F_{\mathrm{w} 2} \cos \gamma=F_{\mathrm{r} 2} \sin \left(\alpha_{2}+\alpha_{1}\right)$

$F_{\mathrm{w} 1} \sin \gamma-F_{\mathrm{w} 2} \sin \gamma=F_{\mathrm{r} 2} \cos \left(\alpha_{2}+\alpha_{1}\right)$,

where $\alpha_{1}$ is the angle between $F_{\mathrm{c}}$ and $F_{\mathrm{r}}, \alpha_{2}$ is the angle between $F_{\mathrm{c}}$ and $x$ axis as shown in Fig. 19.

$\alpha_{2}=\arccos \frac{e_{2}}{\sqrt{e_{1}^{2}+e_{2}^{2}}}$

$\alpha_{1}=\arccos \frac{F_{\mathrm{c}}+e_{1} G \cos \beta / \sqrt{e_{1}^{2}+e_{2}^{2}}}{\left(G^{2} \cos ^{2} \beta+F_{\mathrm{c}}^{2}+2 e_{1} F_{\mathrm{c}} G \cos \beta / \sqrt{e_{1}^{2}+e_{2}^{2}}\right)^{1 / 2}}$.

Combine Eqs. (A4), (A5), (A6), (A8), (A9) into a set of equations, and let the right side of the equations equal 0 , we can get:

$$
\begin{aligned}
& F_{\mathrm{r} 1}+F_{\mathrm{r} 2}-\left(G^{2} \cos ^{2} \beta+F_{\mathrm{c}}^{2}+2 e_{1} G F_{\mathrm{c}} \cos \beta / \sqrt{e_{1}^{2}+e_{2}^{2}}\right)^{1 / 2}=0 \\
& a_{0} F_{\mathrm{r} 1}+\sqrt{e_{1}^{2}+e_{2}^{2}} G \sin \beta-F_{\mathrm{r} 2}\left(L_{6}-L_{5}-a_{0}\right)=0 \\
& F_{\mathrm{w} 1} \cos \gamma+F_{\mathrm{w} 2} \cos \gamma-F_{\mathrm{r} 2} \sin \left(\alpha_{2}+\alpha_{1}\right)=0 \\
& F_{\mathrm{w} 1} \sin \gamma-F_{\mathrm{w} 2} \sin \gamma-F_{\mathrm{r} 2} \cos \left(\alpha_{2}+\alpha_{1}\right)=0 \\
& G \sin \beta-F_{\mathrm{a} 1}=0
\end{aligned}
$$

\section{Appendix C: Stiffness and damping model at left supporting point}

As shown in Fig. $11, K_{\mathrm{s} 1}$ and $m_{\mathrm{s} 1}$ are the vertical stiffness and mass of front bracket. $K_{\mathrm{b} 1}$ and $C_{\mathrm{b} 1}$ are the radial stiffness and damping of the spherical roller bearing.

When considering the mass $m_{\mathrm{s} 1}$, the composite stiffness of the front bracket is (Wen et al., 1999):

$$
K_{\mathrm{s} 1}{ }^{\prime}=K_{\mathrm{s} 1}-m_{\mathrm{s} 1} \omega^{2}
$$

The vertical stiffness $K_{\mathrm{s} 1}{ }^{\prime}$ should be changed into $K_{\mathrm{s} 1}^{\mathrm{c}}$, the direction of which is perpendicular to the mixing drum's axial direction:

$K_{\mathrm{s} 1}^{\mathrm{c}}=K_{\mathrm{s} 1}{ }^{\prime} / \cos ^{2} \beta$.

The composite stiffness $K_{\mathrm{s} 1}^{\mathrm{c}}$ of the front bracket and the radial stiffness $K_{\mathrm{b} 1}$ of the bearing are in series:

$$
K_{\text {left }}=\left(\frac{1}{K_{\mathrm{b} 1}}+\frac{1}{K_{\mathrm{s} 1}^{\mathrm{c}}}\right)^{-1} .
$$

Bring Eqs. (A13) and (A14) into Eq. (A15), we can get:

$$
K_{\text {left }}=\frac{K_{\mathrm{b} 1}\left(K_{\mathrm{s} 1}-m_{\mathrm{s} 1} \omega^{2}\right) / \cos ^{2} \beta}{K_{\mathrm{b} 1}+\left(K_{\mathrm{s} 1}-m_{\mathrm{s} 1} \omega^{2}\right) / \cos ^{2} \beta} .
$$


Competing interests. The authors declare that they have no conflict of interest.

Acknowledgements. The authors would like to thank Shanghai Electric Hydraulics \& Pneumatics Co., Ltd. for their research cooperation and suggestions. The authors are sincerely grateful to honorable reviewers for their valuable review comments, which substantially improved the article.

Edited by: Lotfi Romdhane

Reviewed by: four anonymous referees

\section{References}

Aleyaasin, M., Ebrahimi, M., and Whalley, R.: Vibration analysis of distributed-lumped rotor systems. Comput. Method. Appl. M., 189, 545-558, 2000.

Dukkipati, R. V., Rao, M. A., and Bhat, R.,: Computer aided analysis and design of machine elements, New Age International, New Delhi, India, 128-131, 2000.

Gao, Y., Li, Z. M., Wang, J. W., Li, X. L., and An, Q. : Influences of bearing housing deflection on vibration performance of cylinder roller bearing-rotor system, P. I. Mech. Eng. K-J. Mul., 227, 106114, 2013.

Gao, Y. D., Ma, Y. Z., and Kong, X. G.: Analysis and Optimization on the Front Supporter of Concrete Mixer Truck, Appl. Mech. Mater., 385-386, 288-291, 2013.

Hamrock, B. J. and Dowson, D.: Ball bearing lubrication, Wiley Publications, New York, USA, 68-69, 1981.

Harsha, S. P.: Non-linear dynamic response of a balanced rotor supported on rolling element bearings, Mech. Syst. Signal Pr., 19, 551-578, 2005

Houpert, L.: An Engineering Approach to Hertzian Contact Elasticity - Part I, J. Tribol.-T. ASME, 123, 582-588, 2001.

Kabus, S., Hansen, M. R., and Mouritsen, O. Ø.: A new quasi-static multi-degree of freedom tapered roller bearing model to accurately consider non-Hertzian contact pressures in time-domain simulations, P. I. Mech. Eng. K-J. Mul., 228, 111-125, 2014.

Li, B. Q.: Rollover prevention technology research of concrete mixer truck based on transport conditions, M.S. thesis, Chongqing Jiaotong University, China, 2013.

Li, L. X., Jiang, Y. Y., Li, Z. M., Zhan, Y. B., and An, Q.: Study on the vibration characteristics of a roller-supporting horizontal centrifugal casting machine, P. I. Mech. Eng. K-J. Mul., 230, 99109, 2016.
Li, R. M., Zhang Y. C., Yu, Y. X., Li. Y., and He, Y. Y.: Research and application of load of concrete mixer truck special reducer, Journal of Mechanical Transmission, 37, 27-31, 2013.

Liu, Z. S., Qian, D. S., Sun, L. Q., and Wang, Y. L.: Stability analyses of inclined rotor bearing system based on non-linear oil film force models, Proc. I. Mech. Eng. C-J. Mec., 226, 439-453, 2012.

Ma, F. B., Li, Z. M., Wu, B. J., and An, Q.: An accurate calculation method for heat generation rate in grease-lubricated spherical roller bearing, P. I. Mech. Eng. J-J. Eng., 230, 472-480, 2016.

Matsubara, M., Rahnejat, H., and Gohar, R.: Computational modeling of precision spindles supported by ball bearings, Int. J. Mach. Tool. Manu., 28, 429-442, 1988.

Matthew, C. and Sergei, G.: Nonlinear dynamic behaviour of vertical and horizontal rotors in compliant liner tilting pad journal bearings: Some design considerations, Tribol. Int., 82, 142-152, 2015.

Qian, D., Liu, Z., Yan, J., Sun, L., and Wang, Y.: Numerical and Experimental Research on Periodic Solution Stability of Inclined Rotor Journal Bearing System, in: ASME 2011 Turbo Expo: Turbine Technical Conference and Exposition, Vancouver, British Columbia, Canada, 6-10 June 2011, Volume 6: Structures and Dynamics, Parts A and B, GT2011-45632, 321-330, https://doi.org/10.1115/GT2011-45632, 2011.

Rahnejat, H. and Gohar, R.: Design of profiled taper roller bearings, Tribol. Int., 12, 269-275, 1979.

Shi, M. L., Wang, D. Z., and Zhang, J. G.: Nonlinear dynamic analysis of a vertical rotor-bearing system, J. Mech. Sci. Technol., 27, 9-19, 2013.

Tsai, C. Y. and Huang, S. C.: Transfer matrix method to vibration analysis of rotors with coupler offsets, Shock Vib., 20, 97-108, 2013.

Wen, B. C., Gu, J. L., Xia, S. B., and Wang, Z.: Advanced rotor dynamics: theory, technology and applications, China Machine Press, Beijing, China, 3-5, 1999.

$\mathrm{Wu}, \mathrm{X}$. J.: Study on ride comfort modeling and analysis of concrete mixer truck, $\mathrm{PhD}$ thesis, Huazhong University of Science and Technology, China, 2011.

Yan, W.: Simulation research of mixer truck at unsteady operating conditions, M.S. thesis, Chongqing Jiaotong University, China, 2013.

Yang, J. P., Zeng, H., Zhu, T. Q., and An, Q.: Study on the dynamic performance of tilt rotor supported by spherical roller bearing, P. I. Mech. Eng. K-J. Mul., 231, 156-166, https://doi.org/10.1177/1464419316655956, 2016. 